\title{
浅析大数据技术在水利工程信息化建设中的运用
}

刘小利

\author{
山东金桥建设项目管理有限公司，山东潍坊 262700
}

[摘要]在我们国家, 水利工程的受重视程度是较高的, 从上世纪 90 年代起就开始展开信息化建设工作, 然而当时的信息技术 水平是较低的，所以只能够对信息进行存储、交流，以及制作二维图。在现阶段，信息化管理发生了很大的转变，通过其可 以实现数据共享, 并为决策提供服务, 如此就可保证水利工程管理的实效性大幅提高。随着信息技术发展速度持续加快, 互 联网技术、云计算技术、物联网技术等开始得到应用, 这就使得数据量大幅增加, 进而保证水利工程信息化建设工作能够顺 利展开。

[关键词]大数据技术; 水利工程; 信息化建设

DOI：10.33142/sca.v4i3.4027 中图分类号：TV21-39 文献标识码：A

\section{Brief Analysis on Application of Big Data Technology in Water Conservancy Project Informatization Construction}

LIU Xiaoli

Shandong Jinqiao Construction Project Management Co., Ltd., Weifang, Shandong, 262700, China

\begin{abstract}
In our country, the importance of water conservancy projects is high. Since the 1990s, information construction has been carried out. However, the level of information technology at that time was low, so it was only able to store and exchange information and make two-dimensional maps. At the present stage, great changes have taken place in information management, through which data sharing can be realized and decision-making services can be provided, so as to ensure the effectiveness of water conservancy project management greatly improved. With the development of information technology continues to accelerate, Internet technology, cloud computing technology, Internet of things technology began to be applied, which makes the amount of data increased significantly, so as to ensure the smooth development of water conservancy project information construction.
\end{abstract}

Keywords: big data technology; water conservancy project; information construction

\section{引言}

在当前时期, 国内经济呈现出较快的发展趋势, 大家的物质生活水平有了很大的改变, 对基础设施的关注度也明 显提高。从水利工程项目来看, 若想保证广大群众的内在需要得到满足, 应该要对建设质量予以重点关注, 而要实现 这个目标, 施工过程中则要对现代信息技术加以充分利用, 特别是要将大数据技术具有的实际价值展现出来, 如此方 可使得水利工程建设的信息化程度大幅提高, 保证工程建设有序展开, 并能够为大家的生活提供有价值服务。

\section{1 大数据技术和水利大数据概述}

\section{1 大数据技术}

所谓大数据技术, 简单来说就是对海量数据进行收集、分析, 并发挥出参考作用, 使得目标任务能够顺利达成。 此种技术的优势是明显的, 通过其能够保证数据分析更加的全面, 可以为决策提供必要的帮助 ${ }^{[1]}$ 。

\section{2 水利大数据}

所谓水利大数据, 即是在展开水利工作时对大数据加以运用, 确保相关人员能够获得所需数据, 确保相关工作能 够顺利展开, 并赋有实效。从传统运营活动来看, 数据收集虽然受到关注, 然而大量信息并不具有实用价值, 而利用 大数据技术则能够切实完成数据筛选, 进而保证数据价值得以充分展现。从当下水利工程建设的实际情况来看, 遥感、 射频、采集等技术的应用是较为常见的, 而这也就使得数据量大幅增加, 而且数据类型是众多的, 管道分布、泵站运 行、地质地貌等均在其内。采用传统手段对这些数据进行分析难度非常大, 结果也难以保证准确, 通过大数据技术则 可以改变此种状况, 而且数据共享目的也能够切实达成, 管理决策过程中也就能够获得必要的数据作为支撑 ${ }^{[2]}$ 。 


\section{2 我国水利工程信息化建设的现状}

\section{1 落后于行业发展需求}

国内经济的发展速度在加快, 对于水利单位来说, 除了要对经济发展予以关注, 同时也要考虑到环境影响、水土 保持等方面的问题。虽然水利工程的信息化建设在深入展开, 然而数据采集采用的方法依然是较为陈旧的, 这对行业 发展造成的影响较大。比方说, 有效水利单位虽然认识到水土保持的重要性, 但是自身的专业能力是较为薄弱的, 这 就使得专业评价的效果不够理想, 尤其是所需的信息得不到满足, 这就导致相关工作难以有效展开, 整个行业的发展 也受到限制 ${ }^{[3]}$ 。

\section{2 上层设计不足}

展开水利工程信息化建设工作时, 一定要保证上层设计能够切实做到位。在展开上层设计时, 必须要从实际情况 出发, 选择最为合适的方法, 如此方可使得设计真正呈现出针对性。这里需要提醒的是, 切不可出现 “一手抓” 现象, 因为其造成的危害是非常大的, 有些单位领导自身的专业能力并不强, 由其制定的总体规划存在不少的漏洞, 上层设 计方案的合理性也明显欠缺, 这对水利工程信息化建设产生的影响非常大, 甚至会导致相关工作根本无法展开。国内 不少的水利单位并没有认识到上层设计的价值所在, 所以设计中出现的问题是较多的, 如果没有能够顺利解决的话, 则会使得水利建设工作难以展开, 效果达不到预期 ${ }^{[4]}$ 。

\section{3 缺乏有效的协调平台}

从水利工程信息化建设的现状来看, 平台协调性这个问题是客观存在的, 如果这一问题未能得到解决, 产生的影 响将是非常大的。随着数据共享进一步加深, 如果平台具有的协调性无法满足需要的话, 工程管理、经营效果就很难 达到预期, 信息价值也得不到体现。我们国家的平台并不是十分协调, 这就使得问题发生概率持续增加, 而且数据管 理机制也难以真正实现统一, 如此一来, 数据异构这样的问题就很难得到消除。一旦相关的单位、部门间未能真正实 现信息共享，必然使得决策工作受到影响。

\section{3 大数据技术在水利工程信息化建设中的应用}

国内的一些水利单位正在大力推进信息化建设工作，并提出了较为完善的建设方案，效果是较为理想的。若想使 得相关的建设工作能够顺利展开, 水利单位必须要对行业发展的现状有清晰的认知, 在此基础上对建设方案予以细化, 确保信息化平台具有的功能更加的全面，尤其是要保证大数据技术的作用能够充分发挥出来。

\section{1 基础与制度建设}

为了保证水利工程信息化建设能够顺利进行，制度支撑是必不可少的。对制度方案进行制定的过程中，单位现状 是不可忽视的, 而且要对其他单位的成熟经验予以借鉴。进入到大数据时代后, 信息化平台成为大家关注的重点, 建 设过程中要保证硬件、软件满足实际需要, 网络也应该确保稳定。单位领导要对此项工作予以大力支持, 硬件基础的 改造工作要切实做到位, 服务器性能应该大幅提高, 并切实完成好虚拟化配置。平台所处网络环境应该要更为安全, 并配置适合的软件、硬件, 在此基础上展开综合管理系统的建设, 确保平台功能得到提升, 除了具备基础功能, 同时 要能够完成数据挖掘以及云计算之类的工作, 将 VPN 系统具有的优势充分展现出来, 如此可以使得整个系统的安全性 大幅提高, 而且应用也更为简便。系统完成了信息采集工作后, 必须要完成好安全评估工作, 将所得结果作为依据展 开容灾系统的建设, 如此可以使得数据信息发生外泄的概率大幅降低 ${ }^{[5]}$ 。另外来说, 水利单位还要组织管理人员参加相 关的培训, 确保其专业技能得到提高, 并能够掌握大数据、云计算等方面的知识, 如此方可保证系统建设工作能够顺 利展开，而且在运行、维护过程中所需的人力资源得到切实满足。

\section{2 综合性信息化管理系统}

在对综合性信息化管理系统进行构建时，要将信息化文件、程序文件的基础作用发挥出来，这样可以保证信息化 管理的整体效率大幅提高。水利单位组建起的开发团队中应该包含技能水平高、实践能力强的工作人员，如此方可使 得生产、管理等任务顺利完成, 进而保证平台功能真正实现集成。当综合性信息化管理系统建成之后, 可将办公、经 营、技术、设计、测绘、档案等模块集成于平台之中, 进而使得信息能够真正实现共享, 如此一来, 大数据就能够得 到更为充分的利用, 相关人员可以对相关的数据进行查阅、比对, 数据信息的利用价值自然就可得到保证, 在进行决 策的过程中就可以获得数据支持, 并对水利工程建设的实际情况有切实的了解, 而且相关人员所要承担的工作会大幅 减少，从整个信息化建设来看，管理质量能够有明显的提高，并带来更为理想的工作效益。 


\section{4 结束语}

总之, 在当前全球化经济背景下, 各行业想要实现有序健康发展, 就需要紧紧围绕时代的发展需求, 充分利用大 数据技术, 建设水利工程信息化项目。这就要求相关单位充分了解社会的发展需要, 顺应时代发展潮流, 重视并加强 大数据技术的应用, 结合水利工程信息化建设现状, 完善信息化平台框架和制度, 建立三维数字化协同平台, 搭建综 合性管理系统, 使水利工程信息化管理水平得到提升, 促进水利行业的可持续健康发展, 也为我国社会主义现代化建 设贡献一份力量。

\section{[参考文献]}

[1]佟保根, 赵智磊. 大数据技术在水利工程信息化建设中的运用 $[\mathrm{J}]$. 电子世界, 2021(2): 71-72.

[2] 谢勇,胡德鹏. 大数据技术在水利工程信息化建设中的运用 [J]. 工程技术研究, 2020,5(17): 104-105.

[3]朱丽丽,林勇. 大数据技术在水利工程信息化建设中的运用 $[\mathrm{J}]$. 工程技术研究, 2020, 5(15): 195-196.

[4]许源. 大数据技术在水利工程信息化建设中的运用研究 $[\mathrm{J}]$. 科技创新导报, 2020,17(13): 41-42.

[5]张文豪,李芯, 陈建. 大数据技术在水利工程信息化建设中的运用 $[\mathrm{J}]$. 科技创新与应用, 2020(6): 177-178.

作者简介: 刘小利 (1983.12-), 毕业于: 山东理工大学, 所学专业: 工程管理, 当前就职单位: 山东金桥建设项目管 理有限公司, 职务: 副总经理, 职称: 副高级职称。 